\title{
Chronic pain in people with HIV: a common comorbidity and threat to quality of life
}

\author{
Victoria J Madden ${ }^{1,2}$, Romy Parker ${ }^{1}$ \& Burel R Goodin*,3 (iD) \\ ${ }^{1}$ Department of Anaesthesia \& Perioperative Medicine, Neuroscience Institute, University of Cape Town, Cape Town, Western \\ Cape, South Africa \\ ${ }^{2}$ HIV Mental Health Research Unit, Department of Psychiatry and Mental Health, Neuroscience Institute, University of Cape Town, \\ Cape Town, Western Cape, South Africa \\ ${ }^{3}$ Department of Psychology, University of Alabama at Birmingham, Birmingham, AL 35294, USA \\ *Author for correspondence: Tel.: +1 205934 6536; Fax: +1 205975 6110; bgoodin1@uab.edu
}

\section{Practice points}

- Nonmalignant chronic pain is very common in people with HIV (PWH). Clinical epidemiology research suggests that chronic pain prevalence rates are higher in PWH than in the general population.

- Painful distal peripheral neuropathy due to HIV itself and early antiretroviral medications was historically considered the primary pain condition in this population; however, PWH contend with a preponderance of non-neuropathic, multi-site chronic pain of unknown etiology at present.

- In the USA, the risk of opioid analgesic misuse is elevated in PWH. Current consensus guidelines do not recommend opioid analgesics as a first-line treatment for the long-term management of chronic pain in PWH.

- Suboptimal treatment adherence and retention in care are more frequent in PWH when they also have a comorbid chronic pain condition.

- Current research suggests that persistent immune activation may influence the development and maintenance of chronic pain in $\mathrm{PWH}$.

- Stigma due to HIV and chronic pain may interact to compromise health-promoting behaviors such as medication adherence, retention in care and good patient-provider relationships.

- PWH and chronic pain report more mood disturbances than PWH who do not have chronic pain, and many explicitly identify psychosocial factors as strong influences over their pain.

- The future of improved care for PWH and chronic pain depends heavily on internationally collaborative, adequately funded and strategically implemented research efforts, as well as the careful incorporation of research findings into better treatments and training of clinicians.

Evidence indicates that over half of all people with HIV (PWH) will experience nonmalignant chronic pain throughout their lifetimes, with increasing prevalence as they age. Peripheral neuropathy resulting from the neurotoxic effects of HIV itself and the medications used to treat HIV were widely considered the primary cause of acute and chronic pain early on in the antiretroviral treatment era. However, recent studies suggest a predominance of non-neuropathic (e.g., musculoskeletal) pain in PWH with uncertain etiology. Chronic pain is often widespread in $\mathrm{PWH}$, affecting multiple body locations. Additional research is needed to better understand contributors to chronic pain in $\mathrm{PWH}$, which is likely to include biological (e.g., immune dysregulation), psychological (e.g., substance abuse) and social (e.g., stigma) factors.

First draft submitted: 27 January 2020; Accepted for publication: 16 April 2020; Published online: 2 June 2020

Keywords: access to care $\bullet$ biopsychosocial $\bullet$ HIV $\bullet$ inflammation $\bullet$ mental health $\bullet$ pain $\bullet$ prevalence $\bullet$ quality of life • social support • stigma

Nonmalignant chronic pain is increasingly recognized in clinical and research communities as a common comorbidity among people living with the HIV. Chronic pain generally refers to pain that has persisted for at least three consecutive months, and that has been present on at least half the days in the past 6 months. Chronic pain is widely prevalent in people with HIV (PWH), and a direct threat to their quality of life. Indeed, PWH consistently identify chronic pain as a health priority to be addressed as part of their routine HIV care [1]. Why PWH appear to be at greater risk of developing chronic pain relative to people without HIV remains unclear. Further, etiological 
contributors to chronic pain development and maintenance in PWH is understudied, which has impeded the development of an appropriate evidence base to inform best practices for pain management in this population. The objective of this special report is to provide a brief overview of this emerging field, while also highlighting important areas for future research. Our ultimate goal is to help stimulate new lines of investigation that will promote effective clinical care of PWH with chronic pain.

\section{Chronically painful HIV-associated peripheral neuropathy}

HIV was first identified as the cause of AIDS in 1983. At that time, the prominent clinical features of HIV infection were immunosuppression with associated lymphadenopathy and susceptibility to opportunistic infections. Pain reported by PWH was believed to usually have an identifiable and treatable cause, such as an infectious or neoplastic lesion [2]. The transition to AIDS and subsequent death was quick; therefore, most chronic pain in PWH was treated with an 'end-of-life' approach similar to that used in the management of pain in terminal cancer [3]. The goal of most HIV research was to identify a cure for HIV. Antiretroviral medications were an important breakthrough in this search for a cure. Although these medications do not eliminate the virus entirely, they limit its replication; thus allowing some recovery of immune function and markedly delaying the progression of HIV to AIDS and death $[4,5]$. However, the early antiretroviral medications were associated with particularly adverse effects, including a distal sensory polyneuropathy that frequently rendered patients unable to walk due to intense pain and paresthesia upon weight bearing. This neuropathic pain was arguably the first clearly characterized 'chronic pain syndrome' to be linked directly to HIV and HIV care [6,7]. A flurry of research on how best to prevent and treat this HIV-related chronic neuropathic pain followed, with substantial support from academic and industry research bodies [8]. It became clear that certain early antiretroviral medications (particularly nucleoside reverse transcriptase inhibitors including stavudine, didanosine and zalcitabine) were associated with peripheral neuropathy and related chronic pain $[7,9]$. Clinicians and researchers were trained to detect neuropathy early and to switch affected patients to a different antiretroviral medication to limit medication-induced peripheral neural toxicity [10]. Although chronic pain associated with HIV sensory neuropathy remains a clinical challenge for PWH today, efforts in research and advocacy have vastly improved awareness of the problem while providing clear pathways of action for clinicians.

\section{Beyond chronically painful distal neuropathy in PWH}

As antiretroviral treatment pathways have improved, so has medication adherence. Successful antiretroviral treatment usually renders the plasma load of HIV undetectable to most assays within 3 months [11]. Thus, in many regions of the world, HIV has become a manageable rather than a fatal disease. Although the data are mixed, recent studies suggest that the prevalence of chronically painful HIV sensory neuropathy is lower in people treated with modern antiretrovirals, which are less neurotoxic [12,13]. However, despite improved disease control and safer antiretrovirals, pain remains a prominent concern with over half of PWH suffering from chronic pain in their lifetimes [14]. In only a small portion does this chronic pain match the well-characterised stocking-and-glove distribution of the distal sensory polyneuropathy that was frequent in the time of the early antiretrovirals. On the contrary, $\mathrm{PWH}$ and chronic pain report a median of 2-5 pain sites each, with the head, abdomen, chest, back and legs being particularly common sites of pain [15-17]. Although some PWH with sensory neuropathy do experience chronic neuropathic pain, a recent analysis of stavudine-unexposed people taking combination antiretroviral treatment found that only $38.5 \%$ of those reporting neuropathic pain symptoms also had clinically diagnosable HIV sensory neuropathy [11]. It seems that neuropathy is just one contributor to the problem of chronic pain in HIV, even when that pain has clear 'neuropathic' characteristics. The focus of current research is now shifting toward other mechanisms that may be at play. The importance of such mechanistic research is underscored by inadequate efforts to treat chronic pain in HIV. Two studies based in the Majority World reported that only 24\% (Nigeria) and 18\% (Thailand) of PWH and chronic pain were receiving adequate pain therapy [18,19]. Three US-based studies asked people taking analgesic therapies to estimate the amount of pain relief their therapies achieved, and reported relief scores in the range of 50-65\% [20-22]. Clearly, better treatments are needed. In fact, in some areas of the world, attempts to treat HIV-related chronic pain seem to have led to more problems than benefits. Reports from the USA indicate that opioid analgesics were historically prescribed in large numbers to PWH and chronic pain [22]. In the USA, the risk of opioid analgesic misuse is elevated in HIV populations, and opioid overdose is a leading cause of accidental death [23]. Although opioid misuse is not as prominent a concern in other countries with large populations of $\mathrm{PWH}$, it is important that any attempts to treat chronic pain in PWH acknowledge that the current consensus guideline does not recommend opioid analgesics as a first-line treatment for the long-term management of chronic 
pain in PWH [24]. Unfortunately, research suggests that very few PWH and chronic pain receive adequate pain relief (i.e., analgesia) from pharmacologic treatments [18].

\section{Current impact}

Chronic pain has widespread negative consequences. It affects not only individuals and their clinical care, but also their social networks, their involvement in society and their ability to make an economic contribution [25]. At an individual level, PWH and chronic pain report more mood problems than PWH who do not have chronic pain [15], and many explicitly identify psychosocial factors as strong influences over their chronic pain [26]. US data identify that PWH who have chronic pain also have higher rates of comorbid mental health disorders [27] and are twice as likely to consider suicide as their pain-free peers [28]. The clinical care of HIV is more likely to be compromised when PWH have chronic pain. Suboptimal treatment adherence and retention in care are more frequent in PWH and chronic pain $[29,30]$, placing them at higher risk of viral rebound and worse long-term health outcomes. Societal interactions also suffer given some PWH and chronic pain report that the pain limits their willingness to engage in social activities [26]. Chronic pain is also linked to lower economic participation: one UK/Ireland-based study found that PWH and chronic pain are less likely than their matched, pain-free peers to be working full-time, and more likely to be unemployed or on sick leave [30]. Despite this growing body of evidence demonstrating the widespread negative consequences of chronic pain in $\mathrm{PWH}$, the problem remains largely underappreciated and undertreated. There is a clear need for greater research attention and clinical effort to address chronic pain in PWH.

\section{Current understanding of chronic pain in PWH \& future directions}

One important matter that requires clarification is why chronic pain seems to be more prevalent in $\mathrm{PWH}$ than in people without HIV. In the general (i.e., non-HIV) population, a recent systematic review suggested that approximately $34 \%$ of people globally suffer from chronic pain [31]. By comparison, over half of PWH suffer from chronic pain in their lifetime, and this prevalence increases to $83 \%$ in some populations [14]. It may be that PWH have more (and different) etiological risk factors for chronic pain development than the general population; therefore, chronic pain prevalence rates are higher in PWH. Below, we identify several risk factors - including interacting biological, psychological and social processes - that could underlie the additional burden of chronic pain in PWH compared with the general population. Our list is not exhaustive but may serve to guide future research in this area.

Tissue damage cause by HIV-related co-infections

Although many of the systemic consequences of HIV infection are well controlled by modern antiretroviral treatment, it remains important to acknowledge the possible role of co-infections to which those with HIV are vulnerable. HIV-related opportunistic co-infections can be overt or subclinical. Clinically obvious co-infections would likely be promptly recognized, treated and eliminated - but may leave behind residual tissue damage that continues to contribute to chronic pain. Subclinical infectious processes can go undetected and contribute to chronic pain over longer periods of time, with a greater risk of occult damage to bodily tissue. An example of such a destructive co-infection is tuberculous infection of the spine, which can cause permanent neural damage and promote chronic pain [32]. Co-infection with both tuberculosis and HIV is particularly common in sub-Saharan Africa [33].

\section{Self-monitoring for physical symptoms}

It is also relevant to acknowledge that the possibility of a subclinical co-infection is an ongoing threat for PWH, and that bodily threat is an important contributor to other processes, such as hypervigilance to physical symptoms, that may increase the risk of pain chronicity [34]. It is possible that PWH are prone to engage in self-monitoring for physical symptoms, including pain, as an indicator of co-infection or worsening HIV disease. Although such vigilance may be necessary at times, there is a risk that PWH may develop an enhanced state of sensory sensitivity accompanied by exaggerated scanning behaviors, or searches for threatening information (i.e., hypervigilance). The common result of such hypervigilance for physical symptoms is that normal, everyday sensory experiences are gradually misinterpreted as threatening and may contribute to more perceived pain [35]. In this way, the attentional bias toward physical symptoms is misinterpreted as painful results in increased interference with daily living. Although there is a paucity of research on such attentional or cognitive biases in the context of HIV, there is a 
growing body of literature on such biases in survivors of cancer, who also live with the constant threat of disease recurrence [36].

\section{Deficits in social support}

HIV is associated with a host of negative social circumstances. PWH typically have less stable or narrower social support networks than those without HIV [37]. The fact that HIV is a lifelong disease that must be managed with daily medication and frequent healthcare visits may contribute to this problem. Plenty of literature describes the psychological and social consequences of managing chronic illness, including pain. The unrelenting and frequently shifting goals of self-management (e.g., medication adherence, stigma management, etc.) represent an additional life stressor that may result in psychological or psychiatric comorbidity and reduced engagement with other important life roles, such as work and socializing [38]. A narrowed focus on HIV self-management may further impoverish a previously rich range of social and work engagements. Thereby resulting in increased attention to personal health-related concerns and fears without the social support that might otherwise allay such worries and foster rapid recovery.

\section{Dysregulated immune activity \& inflammation}

Current research suggests that persistent immune activation may influence the development and maintenance of chronic pain in PWH [39]. HIV infection prompts a set of cytotoxic immune processes, and HIV itself contains elements that can influence sensitivity to pain $[40,41]$. Research in animals and humans has shown that HIV envelope glycoproteins, such as GP120, promote allodynia and hyperalgesia [40]. These HIV glycoproteins can directly invade the afferent neurons of the dorsal root ganglion, resulting in axonal injury and neuronal sensitization [40]. Animals injected with GP120 demonstrate increased HIV-related pain behaviors, possibly due to altered glial activity in the spinal cord dorsal horn [40]. Markers of immune activation, inflammation and coagulation typically decline during suppressive antiretroviral therapy, but remain abnormally elevated in many HIV-infected individuals and predict subsequent mortality and non-AIDS morbidities [41]. Further, PWH and active viremia often demonstrate markedly increased systemic inflammation and exaggerated inflammatory responses to stressful stimuli [42]. HIVmediated destruction of gut mucosa may contribute to this by allowing the translocation of microbial products that promote local and systemic inflammation [43]. Increased inflammation in PWH is likely related to several common negative HIV-related health outcomes including cardiovascular disease, co-infections, cancer, Type II diabetes and frailty. Several small cohort trials suggest that reducing chronic inflammation may yield important improvements in these comorbidities for PWH [41]. In largely non-HIV populations, high levels of inflammation promote heightened sensitivity to painful stimuli and poor chronic pain outcomes, including greater clinical pain severity and worse physical functioning [43]. These findings are not yet extrapolated to $\mathrm{PWH}$, but they suggest that chronic inflammation may also contribute to neuronal sensitization, chronic pain development and worse clinical pain outcomes in this population.

\section{HIV \& chronic pain stigma}

For PWH, HIV-related stigma is linked to a remarkable number of negative outcomes in the psychological, social and physical health domains, which interactively promote poor quality of life [44]. Chronic pain is also known to be directly linked to stigma, especially when the chronic pain is medically unexplained [45]. HIV and chronic pain stigma may interact to compromise health-promoting behaviors such as medication adherence, retention in care and patient-provider relationships [44]. It also appears that people experiencing stigmatization are at risk for developing more severe and persistent pain [45]. When stigmatized, PWH often experience social rejection, isolation and social pain that may lead to increased pain sensitivity through shared neural networks linking social rejection to pain [46]. Furthermore, recent work has demonstrated that anxiety sensitivity may mediate the relationship between HIV stigma and pain [47]. Anxiety sensitivity refers to a fear of sensations related to arousal, based on the idea that such sensations have negative consequences on well-being. Self-monitoring for sensations related to arousal may foster anxiety sensitivity and, in some people, exacerbate the enhancing effect of stigma on pain.

\section{Reduced access to services}

PWH may have difficulty accessing healthcare due to barriers including stigma, concerns about confidentiality, lack of finances or the simple absence of geographically accessible services [48]. Many systems that serve PWH are overburdened, such that chronic pain may be de-prioritized from among the wide range of issues to be addressed 
during a healthcare visit [49]. This may be even more likely to occur in hierarchical healthcare systems and when disempowerment or lack of education leaves the person with HIV ill-equipped to draw the clinician's attention to their chronic pain. In such situations, failure to access early care for a painful condition may increase the risk of pain chronicity.

\section{Shared risk factors for contracting HIV \& developing chronic pain}

A host of individual and contextual features may represent dual risk for both HIV and chronic pain. It is critical to recognize that HIV is not contracted in a socio-economic vacuum. Adverse childhood experiences, social deprivation and psychiatric illness may represent dual elevated risk for both contracting HIV and developing chronic pain. Others have tentatively proposed that the apparently dramatic rate of neurocognitive disorders in PWH may actually reflect a higher base rate of poor performance on neurocognitive testing among people whose early development occurred under socially deprived circumstances, rather than an effect of having HIV itself [50]. This proposal would be supported if data revealed equivalent performance on neurocognitive testing by people with and without HIV who grew up in similarly deprived circumstances - a question that, to our knowledge, has yet to be examined. Moreover, we speculate that a similar possibility exists in the context of chronic pain and HIV. Specifically, that the greater prevalence of chronic pain in PWH may actually reflect a higher base rate of other biopsychosocial factors that carry risk for chronic pain, rather than a direct influence of HIV itself. Additional research is needed to better elucidate the extent to which chronic pain development in PWH is specific to HIV, and/or related to other factors that increase risk for contracting HIV as well as developing chronic pain.

\section{Future perspective}

Future research is needed for better understanding of chronic pain risk factors that are either causatively linked to HIV itself or to the consequences of having HIV. Such research has the potential to move the field forward by providing a logical rationale for the focused development of treatments. That is, treatments could be developed to address specific biopsychosocial contributors to chronic pain. If such treatments are successful in reducing or removing these contributors, they should also reduce chronic pain for PWH. Future research on this topic may also help elucidate the reasons why chronic pain appears to be much more prevalent in PWH compared with the general (non-HIV) population.

One obvious barrier to progress in chronic pain management in HIV is that many organizations that fund research have been slow to prioritize questions of ongoing health and quality of life for PWH. The history of the epidemic is such that much of the funding focus has remained on strategies to prevent transmission and the search for a cure, even while millions of people with 'controlled HIV disease' face a long life with chronic pain and diminished quality of life [51]. In sub-Saharan Africa, home to the largest population of PWH in the world, funding organizations largely remain focused on curative or life-saving research. However, selected reports and a recent funding call from the United States National Institutes of Health for multinational research in noncommunicable diseases that co-exist with HIV indicates a possible shift in this funding deficit, which is welcome [52,53]. Sustainable, multinational funding support will be crucial for the vast, multinational problem of HIV-related chronic pain to be systematically and effectively addressed. The fact HIV clinicians are typically not (or inadequately) taught to assess or treat chronic pain represents a second barrier to progress in our understanding of pain in HIV.

Considering the worldwide scale of the HIV epidemic and the burden of chronic pain in PWH, future progress will require multinational, interdisciplinary partnerships to understand the complexity of chronic pain in HIV and its impact. Effective treatments can only be developed and tested in a systematic way when the problem that is to be treated - chronic pain in HIV - is thoroughly understood. Previously, most research on chronic pain in HIV has happened in silos of professional disciplines or geographical locations. However, the recently formed Global Task Force on pain in HIV is envisioned to change this by fostering partnerships between patients, clinicians and researchers from diverse locations, cultural groups and disciplines [54].

The future of improved care for PWH and chronic pain depends heavily on internationally collaborative, adequately funded and strategically implemented research efforts, as well as the careful dissemination of research findings into better training of clinicians and empowerment of PWH. It will be critical to assess the impact of these efforts on chronic pain outcomes that are relevant and valuable to PWH (from varied contexts and backgrounds), and to adapt the efforts according to what these assessments reveal. None of these steps are simple or quickly implemented, but each will be necessary to achieve the goal of reducing chronic pain and its widespread negative consequences for PWH. 
Funding \& competing interests disclosure

Support for this publication was provided by the National Heart, Lung and Blood Institute of the National Institutes of Health under award number R01HL147603 (BR Goodin). Additional support was provided by the Fogarty International Center of the National Institutes of Health under award K43TW011442 (VJ Madden). The authors have no other relevant affiliations or financial involvement with any organization or entity with a financial interest in or financial conflict with the subject matter or materials discussed in the manuscript apart from those disclosed.

No writing assistance was utilized in the production of this manuscript.

Open access

This work is licensed under the Attribution-NonCommercial-NoDerivatives 4.0 Unported License. To view a copy of this license, visit http://creativecommons.org/licenses/by-nc-nd/4.0/

\section{References}

Papers of special note have been highlighted as: $\bullet$ of interest; $\bullet \bullet$ of considerable interest

1. Bristowe $\mathrm{K}$, Clift P, James R et al. Towards person-centred care for people living with HIV: what core outcomes matter, and how might we assess them? A cross-national multi-centre qualitative study with key stakeholders. HIV Med. 20, 542-554 (2019).

2. O'Neill WM, Sherrard JS. Pain in human immunodeficiency virus disease: a review. Pain 54(1), 3-14 (1993).

3. Poorolajal J, Hooshmand E, Mahjub H, Esmailnasab N, Jenabi E. Survival rate of AIDS disease and mortality in HIV-infected patients: a meta-analysis. Public Health. 139, 3-12 (2016).

4. Cohen OJ, Fauci AS. HIV/AIDS in 1998 - gaining the upper hand? JAMA 280(1), 87-88 (1998).

5. Lipkin WI, Parry G, Kiprov D, Abrams D. Inflammatory neuroprithy in homosexual men with lymphadenopathy. Neurology $35(10)$, 1479-1479 (1985).

6. Yarchoan R, Thomas R, Allain J-P et al. Phase I studies of $2^{\prime}, 3^{\prime}$-dideoxycytidine in severe human immunodeficiency virus infection as a single agent and alternating with zidovudine (AZT). Lancet 331(8577), 76-81 (1988).

7. Phillips TJC, Cherry CL, Cox S, Marshall SJ, Rice ASC. Pharmacological treatment of painful HIV-associated sensory neuropathy: a systematic review and meta-analysis of randomised controlled trials. PLoS ONE 5(12), e14433 (2011).

8. Blum AS, Dal Pan GJ, Feinberg J et al. Low-dose zalcitabine-related toxic neuropathy: frequency, natural history, and risk factors. Neurology 46(4), 999-1003 (1996).

9. WHO. Scaling up Anti-retroviral Therapy in Resource-limited Settings: Treatment Guidelines for a Public Health Approach. Geneva, Switzerland (2003). www.who.int/3by5/publications/documents/arv_guidelines/en/

10. Maartens G, Celum C, Lewin SR. HIV infection: epidemiology, pathogenesis, treatment, and prevention. Lancet 384(9939), 258-271 (2014).

11. Octaviana F, Safri AY, Setiawan DD et al. Neuropathic pain in HIV patients receiving ART without stavudine in an Indonesia Referral Hospital. J. Neurol. Sci. 397, 146-149 (2019).

12. Pillay P, Wadley AL, Cherry CL, Karstaedt AS, Kamerman PR. Clinical diagnosis of sensory neuropathy in HIV patients treated with tenofovir: a 6-month follow-up study. J. Peripher. Nerv. Syst. 24(4), 304-313 (2019).

13. Vecchio AC, Marra CM, Schouten J et al. Distal sensory peripheral neuropathy in human immunodeficiency virus Type 1-positive individuals before and after antiretroviral therapy initiation in diverse resource-limited settings. Clin. Infect.

Dis. doi:10.1093/cid/ciz745 (2019) (Epub ahead of print).

- This multinational longitudinal study shows that there was substantial prevalence of distal sensory peripheral neuropathy (DSPN) in people living with HIV (PLWH) who had not previously been initiated on a combined antiretroviral regimen (cART). Once intiated on a cART regiment, the overall prevalence of DSPN among PLWH decreased after virologic suppression was achieved.

14. Parker R, Stein DJ, Jelsma J. Pain in people living with HIV/AIDS: a systematic review. J. Int. AIDS Soc. 17, 18719 (2014).

15. Namisango E, Harding R, Atuhaire L et al. Pain among ambulatory HIV/AIDS patients: multicenter study of prevalence, intensity, associated factors, and effect. J. Pain 13(7), 704-713 (2012).

16. Peltzer K, Phaswana-Mafuya N. The symptom experience of people living with HIV and AIDS in the Eastern Cape, South Africa. BMC Health Serv. Res. 8, 271 (2008).

17. Parker R, Jelsma J, Stein DJ. Pain in amaXhosa women living with HIV/AIDS: a cross-sectional study of ambulant outpatients. $B M C$ Womens Health. 17(1), 31 (2017).

18. Robbins NM, Chaiklang K, Supparatpinyo K. Undertreatment of pain in HIV+ adults in Thailand. J. Pain Symptom Manage. 45(6), 1061-1072 (2013).

19. Ebirim LN, Otokwala JG. Inadequate pain relief in ambulatory patients with human immunodeficiency virus disease in Port Harcourt. HIV/AIDS (Auckland, N.Z.). 5, 199-203 (2013). 
20. McCormack JP, Li R, Zarowny D, Singer J. Inadequate treatment of pain in ambulatory HIV patients. Clin. J. Pain 9(4), 279-283 (1993).

21. Merlin JS, Cen L, Praestgaard A et al. Pain and physical and psychological symptoms in ambulatory HIV patients in the current treatment era. J. Pain Symptom Manage. 43(3), 638-645 (2012).

22. Edelman EJ, Gordon K, Becker WC et al. Receipt of opioid analgesics by HIV-infected and uninfected patients. J. Gen. Internal Med. 28(1), 82-90 (2013).

23. Cunningham CO. Opioids and HIV infection: from pain management to addiction treatment. Top. Antivir. Med. 25(4), 143-146 (2018).

24. Bruce RD, Merlin J, Lum PJ et al. 2017 HIVMA of IDSA clinical practice guideline for the management of chronic pain in patients living with HIV. Clin. Infect. Dis. 65(10), e1-e37 (2017).

-. This clinical practice guideline provides recommendations for the management and treatment of chronic pain in people living with HIV. The quality of evidence and strength of recommendations are provided according to the GRADE (grading of recommendations, assessment, development and evaulatuion) methodology.

25. Breivik H, Eisenberg E, O'Brien T. The individual and societal burden of chronic pain in Europe: the case for strategic prioritisation and action to improve knowledge and availability of appropriate care. BMC Public Health 13(1), 1229 (2013).

26. Scott W, Arkuter C, Kioskli K et al. Psychosocial factors associated with persistent pain in people with HIV: a systematic review with meta-analysis. Pain 159(12), 2461-2476 (2018).

-. This systematic review and meta-analysis reveal that pain outcomes in people with HIV (PWH) were associated with psychosocial factors including: depression, psychological distress, post-traumatic stress, drug abuse, sleep disturbance, reduced antiretroviral adherence, health care use, missed HIV clinic visits, unemployment and stigma.

27. Merlin JS, Westfall AO, Raper JL et al. Pain, mood, and substance abuse in HIV: implications for clinic visit utilization, antiretroviral therapy adherence, and virologic failure. J. Acquir. Immune Defic. Syndr. 61(2), 164-170 (2012).

28. Marcus KS, Kerns RD, Rosenfeld B, Breitbart W. HIV/AIDS-related pain as a chronic pain condition: implications of a biopsychosocial model for comprehensive assessment and effective management. Pain Med. 1(3), 260-273 (2000).

29. Denis CM, Morales KH, Wu Q, Metzger DS, Cheatle MD. Association between diagnoses of chronic noncancer pain, substance use disorder, and HIV-related outcomes in people living with HIV. J. Acquir. Immune Defic. Syndr. 82, S142-S147 (2019).

- In this US-based study, there was significant overlap between chronic pain, psychiatric diagnoses and substance use disorder in PWH. Chronic pain preceded the onset of psychiatric comorbidity and substance use disorder is a substantial proportion of individuals; opioids for pain management may have been an inciting factor.

30. Sabin CA, Harding R, Bagkeris E et al. Pain in people living with HIV and its association with healthcare resource use, well being and functional status. AIDS (London, England). 32(18), 2697-2706 (2018).

31. Jackson T, Thomas S, Stabile V, Shotwell M, Han X, McQueen K. A systematic review and meta-analysis of the global burden of chronic pain without clear etiology in low-and middle-income countries: trends in heterogeneous data and a proposal for new assessment methods. Anesth. Analg. 123(3), 739-748 (2016).

32. Garg RK, Somvanshi DS. Spinal tuberculosis: a review. J. Spinal Cord Med. 34(5), 440-454 (2011).

33. Naidoo P, Theron G, Rangaka MX et al. The South African tuberculosis care cascade: estimated losses and methodological challenges. J. Infect. Dis. 216(Suppl. 7), S702-S713 (2017).

34. Todd J, Sharpe L, Johnson A, Nicholson Perry K, Colagiuri B, Dear BF. Towards a new model of attentional biases in the development, maintenance, and management of pain. Pain 156(9), 1589-1600 (2015).

35. Crombez G, Van Damme S, Eccleston C. Hypervigilance to pain: an experimental and clinical analysis. Pain 116(1), 4-7 (2005).

36. Heathcote LC, Eccleston C. Pain and cancer survival: a cognitive-affective model of symptom appraisal and the uncertain threat of disease recurrence. Pain 158(7), 1187-1191 (2017).

37. Bravo P, Edwards A, Rollnick S, Elwyn G. Tough decisions faced by people living with HIV: a literature review of psychosocial problems. AIDS Rev. 12(2), 76-88 (2010).

38. Swendeman D, Ingram BL, Rotheram-Borus MJ. Common elements in self-management of HIV and other chronic illnesses: an integrative framework. AIDS Care 21(10), 1321-1334 (2009).

39. Shi Y, Gelman BB, Lisinicchia JG, Tang SJ. Chronic-pain-associated astrocytic reaction in the spinal cord dorsal horn of human immunodeficiency virus-infected patients. J. Neurosci. 32(32), 10833-10840 (2012).

40. Yuan S-B, Shi Y, Chen J et al. Gp120 in the pathogenesis of human immunodeficiency virus-associated pain. Ann. Neurol. 75(6), $837-850$ (2014).

41. Hunt PW. HIV and inflammation: mechanisms and consequences. Curr. HIV/AIDS Rep. 9(2), 139-147 (2012).

42. Tenorio AR, Zheng Y, Bosch RJ et al. Soluble markers of inflammation and coagulation but not T-cell activation predict non-AIDS-defining morbid events during suppressive antiretroviral treatment. J. Infect. Dis. 210(8), 1248-1259 (2014).

43. Ji RR, Chamessian A, Zhang YQ. Pain regulation by non-neuronal cells and inflammation. Science 354(6312), 572-577 (2016). 
44. Rueda S, Mitra S, Chen S et al. Examining the associations between HIV-related stigma and health outcomes in people living with HIV/AIDS: a series of meta-analyses. BMJ Open 6(7), e011453 (2016).

45. De Ruddere L, Craig KD. Understanding stigma and chronic pain: a-state-of-the-art review. Pain 157(8), 1607-1610 (2016).

46. Eisenberger NI, Lieberman MD. Why rejection hurts: a common neural alarm system for physical and social pain. Trends Cogn. Sci. 8(7), 294-300 (2004).

47. Wong CCY, Paulus DJ, Lemaire C et al. Examining HIV-related stigma in relation to pain interference and psychological inflexibility among persons living with HIV/AIDS: the role of anxiety sensitivity. J. HIV AIDS Soc. Serv. 17(1), 1-15 (2018).

- Suggests that the experience of HIV-related stigma may increase pain severity in PWH and chronic pain. Arousal-related sensations misinterpreted as threatening may account for the link between HIV stigma and pain severity.

48. Duff P, Kipp W, Wild TC, Rubaale T, Okech-Ojony J. Barriers to accessing highly active antiretroviral therapy by HIV-positive women attending an antenatal clinic in a regional hospital in western Uganda. J. Int. AIDS Soc. 13(1), 37 (2010).

49. Furber AS, Hodgson IJ, Desclaux A, Mukasa DS. Barriers to better care for people with AIDS in developing countries. BMJ 330(Suppl. S1), 050130 (2005).

50. Nightingale S, Winston A, Letendre S et al. Controversies in HIV-associated neurocognitive disorders. Lancet Neurol. 13(11), 1139-1151 (2014).

51. Lazarus JV, Safreed-Harmon K, Barton SE et al. Beyond viral suppression of HIV-the new quality of life frontier. BMC Med. 14(1), 94 (2016).

-. Building on the UNAIDS 90-90-90 campaign (90\% of PWH diagnosed, on treatment, and virologically suppressed), these authors have proposed a 'fourth 90' which would include greater emphasis on factors that heavily impact quality of life, explicitly including non communicable diseases, mental health and pain.

52. High KP, Brennan-Ing M, Clifford DB et al. HIV and aging: state of knowledge and areas of critical need for research. A report to the NIH Office of AIDS Research by the HIV and Aging Working Group. J. Acquir. Immune Defic. Syndr. 60(Suppl. 1), S1-S18 (2012).

53. Goodenow MM, Gaist P. Importance of HIV/AIDS-related behavioral and social sciences research at the NIH and beyond. J. Acquir. Immune Defic. Syndr. 82(2), S84-S87 (2019).

- The paper address previous, as well as ongoing, efforts to increase behavioral and social sciences research addressing HIV and associated comorbidities. Future research directions are provided related to the application of evidence-based sociobehavioral approaches that could improve HIV clinical end points as well as quality of life.

54. Merlin JS, Hamm M, de Abril Cameron F et al. The Global Task Force for chronic pain in people with HIV (PWH): developing a research agenda in an emerging field. AIDS Care (under review).

-. Chronic pain is a common comorbidity in PWH. Research in this area is growing, but significant gaps remain. A Global Task Force of experts in HIV and chronic pain met and identified clinical and research priorities that must be addressed in the future in order to move the field forward. 